\title{
Developmental cascades linking stress inoculation, arousal regulation, and resilience
}

\section{David M. Lyons *, Karen J. Parker, Maor Katz and Alan F. Schatzberg}

Department of Psychiatry and Behavioral Sciences, Stanford University, Stanford, CA, USA

\section{Edited by:}

Anne Z. Murphy,

Georgia State University, USA

Reviewed by:

Peg McCarthy,

University of Maryland, USA

Tracy L. Bale, University of

Pennsylvania, USA

*Correspondence:

David M. Lyons, Department of

Psychiatry and Behavioral Sciences,

Stanford University, 1201 Welch Rd,

MSLS P104, Stanford, CA 94305-5485,

USA.

e-mail:dmlyons@stanford.edu
Stressful experiences that are challenging but not overwhelming appear to promote the development of arousal regulation and resilience. Variously described in studies of humans as inoculating, steeling, or toughening, the notion that coping with early life stress enhances arousal regulation and resilience is further supported by longitudinal studies of squirrel monkey development. Exposure to early life stress inoculation diminishes subsequent indications of anxiety, increases exploration of novel situations, and decreases stress-levels of cortisol compared to age-matched monkeys raised in undisturbed social groups. Stress inoculation also enhances prefrontal-dependent cognitive control of behavior and increases ventromedial prefrontal cortical volumes. Larger volumes do not reflect increased cortical thickness but instead represent surface area expansion of ventromedial prefrontal cortex. Expansion of ventromedial prefrontal cortex coincides with increased white matter myelination inferred from diffusion tensor magnetic resonance imaging. These findings suggest that early life stress inoculation triggers developmental cascades across multiple domains of adaptive functioning. Prefrontal myelination and cortical expansion induced by the process of coping with stress support broad and enduring trait-like transformations in cognitive, motivational, and emotional aspects of behavior. Implications for programs designed to promote resilience in humans are discussed.

Keywords: emotion regulation, cognitive control, curiosity, cortisol, neuroplasticity, prolonged exposure therapy, resilience

\section{INTRODUCTION}

Adults often cope better with spousal loss, illness, and major accidents if they have previously experienced and coped with stress in childhood (Khoshaba and Maddi, 1999). Work-related stress likewise has fewer deleterious mental health effects in adults previously exposed to work stress in adolescence (Mortimer and Staff, 2004). These and related physiological investigations of children suggest that the process of coping with moderate levels of early life stress promotes the development of arousal regulation and resilience (Ellis et al., 2005; Gunnar et al., 2009). Variously described in studies of humans as inoculating, steeling, or toughening (Garmezy et al., 1984; Dienstbier, 1989; Rutter, 2006), the notion that coping with stress enhances arousal regulation and resilience is further supported by longitudinal studies of squirrel monkey development.

In natural and semi-natural conditions, squirrel monkey mothers and other group members periodically leave newly weaned offspring beginning at 3-6 months of age to forage for food on their own (Boinski and Fragaszy, 1989; Lyons et al., 1998). At this stage of development, offspring are approximately half their adult body size. Initially, brief intermittent separations studied in controlled experimental conditions elicit distress peep-calls and increase plasma levels of cortisol with partial habituation of these measures of arousal observed over repeated separations (Coe et al., 1983; Hennessy, 1986). Later in life, monkeys exposed to repeated intermittent separations show fewer behavioral indications of anxiety, diminished stress-levels of cortisol, and increased sensitivity to glucocorticoid feedback regulation of the hypothalamic-pituitary-adrenal (HPA) axis compared to monkeys not exposed to prior separations (Lyons et al., 1999; Lyons et al., 2000b, Levine and Mody, 2003). These findings are surprising because chronic exposure to severe forms of stress impairs regulation of the HPA axis and increases anxiety in monkeys (Sanchez et al., 2001; O'Connor and Cameron, 2006) whereas intermittent separations that simulate a naturally occurring but stressful condition appear to have opposite effects.

Here we review studies that test the hypothesis that brief intermittent separations are a form of stress inoculation that enhances arousal regulation and resilience. Socially housed squirrel monkeys were randomized to either brief intermittent separations or a non-separated control condition at 17 weeks of age (Parker et al., 2004). For each of 10 total separation sessions, each monkey was removed from the natal group for a 1-h period once a week. In the non-separated control condition, age-matched monkeys remained undisturbed in their natal groups. After completion of these postnatal protocols at 27 weeks of age, all of the monkeys were maintained in identical conditions. Puberty occurs at $2-3$ years of age and the average maximum life span is $\sim 21$ years (Brady, 2000). Behavioral, hormonal, and neuroimaging data were collected at various stages of development as described below. All procedures were conducted in accordance with the NIH Guidelines and were approved by Stanford University's IACUC.

\section{BEHAVIORAL INDICATIONS RESILIENCE}

At 9 months of age, each monkey was placed along with its mother in a novel test environment that contained an assortment of foods and various toy-like objects (Parker et al., 2004). Similar test conditions have been used to demonstrate that severe forms of early 
life stress increase subsequent indications of anxiety in marmoset monkeys and macaques (Hinde and Spencer-Booth, 1971; Andrews and Rosenblum, 1993; Dettling et al., 2002). Here the novel environment test was used to determine whether intermittent separations enhance arousal regulation during forced exposure to an unfamiliar situation.

In the novel environment test, the previously separated and non-separated monkeys were initially similar but differences emerged over repeated test sessions. Gradually, the previously separated monkeys showed fewer signs of anxiety inferred from decreased maternal clinging, increased object exploration, and lower post-test cortisol levels compared to monkeys not exposed to early intermittent separations. As described elsewhere in greater detail (Parker et al., 2004), these results support the suggestion that intermittent separations represent a form of stress inoculation that enhances arousal regulation during forced exposure to a novel situation.

At 1.5 years of age, all monkeys were administered a behavioral test of cognitive control that specifically focused on response inhibition and flexible goal-directed action (Parker et al., 2005). Monkeys were required to inhibit a previously rewarded reaching response in order to efficiently retrieve food treats from a plastic box. In marmoset monkeys and macaques, performance on this test is impaired by lesions of the prefrontal cortex (Diamond, 1990; Dias et al., 1996; Wallis et al., 2001) but not lesions of the hippocampus (Diamond et al., 1989). Test performance is also impaired in a marmoset model of parental neglect (Pryce et al., 2004) and in squirrel monkeys chronically treated with high doses of cortisol (Lyons et al., 2000a). Here we used this test to assess whether intermittent separations improve cognitive control of behavior in the context of changing task-related demands.

The ability to inhibit a previously rewarded behavioral response and flexibly adapt to changing demands gradually improved over repeated test trials. At peak levels of performance, however, all of the previously separated monkeys successfully completed all test trials whereas fewer than half of the monkeys not exposed to intermittent separations achieved similar levels of success (Parker et al., 2005). These and related findings suggest that intermittent separations are a form of stress inoculation that enhances cognitive control of behavior in situations that require flexibility and response inhibition.

At 2.5 years of age, all monkeys were administered a test to examine curiosity in a stress-free condition (Parker et al., 2007). Each monkey was provided free access to explore a familiar or novel toy-like object secured to the wall of a compartment affixed to the monkey's home cage. Similar test conditions have been used to demonstrate that severe forms of early life stress diminish subsequent exploratory tendencies in macaques (Hinde and SpencerBooth, 1971; Sackett, 1972; Roder et al., 1989). Here exploration of novelty was used to assess rearing-related differences in curiosity.

All previously separated and all but one of the non-separated monkeys approached the compartment and subsequently peered inside. More of the previously separated monkeys left their home cage to enter the compartment, and they entered faster, with greater frequency, and spent more time inside the compartment compared to non-separated monkeys. Inside the compartment, more previously separated than non-separated monkeys explored one or both objects, and the previously separated monkeys showed a significant preference for the novel versus familiar object (Parker et al., 2007).

\section{DEVELOPMENTAL CASCADE MODEL OF RESILIENCE}

In the initial novel environment test conducted at 9 months of age, significantly more object exploration was observed in the previously separated compared to non-separated monkeys. Object exploration at 9 months of age was assessed in stressful conditions that involved involuntary removal from the home cage and forced exposure to an unfamiliar situation. In keeping with results from similar studies of forced exposure to novelty in rodents (Hughes, 2007), both previously separated and non-separated monkeys had significantly higher cortisol levels after forced exposure to the novel test environment compared to baseline levels measured in undisturbed home cage conditions (Parker et al., 2004). Moreover, post-test cortisol levels were significantly higher in the non-separated compared to the previously separated monkeys so that differences in object exploration were confounded with physiological indications of anxiety.

In the study conducted at 2.5 years of age, exploratory behavior was assessed by providing free access to a novel test compartment that was connected to the home cage. To further reduce the potentially stressful nature of the test situation, all monkeys were acclimated to the test procedures prior to the start of the study. Post-test cortisol levels were not significantly different from baseline levels measured in undisturbed home cage conditions at 2.5 years of age (Parker et al., 2007). Moreover, neither baseline nor post-test cortisol levels correlated with exploratory behavior in the stress-free test condition at 2.5 years of age. Individual differences previously observed in object exploration at 9 months of age were, however, correlated with differences in test compartment entries and total time spent inside the compartment at 2.5 years of age. Individual differences previously observed in cognitive control at 1.5 years of age also correlated with test compartment entries and total time spent inside the compartment at 2.5 years of age (Lyons and Parker, 2007).

Taken together these findings suggest that brief intermittent separations that simulate a naturally occurring but stressful condition promote enduring trait-like transformations in multiple domains of adaptive functioning. Stress inoculation-induced arousal regulation enhances curiosity inferred from increased exploration of novel situations (Parker et al., 2004). Curiosity reflects more than diminished anxiety because the previously separated monkeys are more curious than non-separated monkeys even in the absence of physiological indications of anxiety (Parker et al., 2007). Curiosity is not an indication of impulsivity because response inhibition is greater in monkeys exposed to intermittent separations compared to non-separated controls (Parker et al., 2005). Curiosity motivates individuals to seek new opportunities for action (Silvia, 2008). Engagement in new situations that entail challenging but not overwhelmingly stressful experiences have cascading effects that promote further adaptations in cognitive, motivational, and socioemotional aspects of behavior (Mason, 1971; Gibson, 1988).

Based on these considerations we believe that the process of coping with early life stress enhances arousal regulation which promotes curiosity and thereby amplifies the effects induced by coping with early life stress (Figure 1). The initial step of "programming" arousal regulation in response to early life stress may 


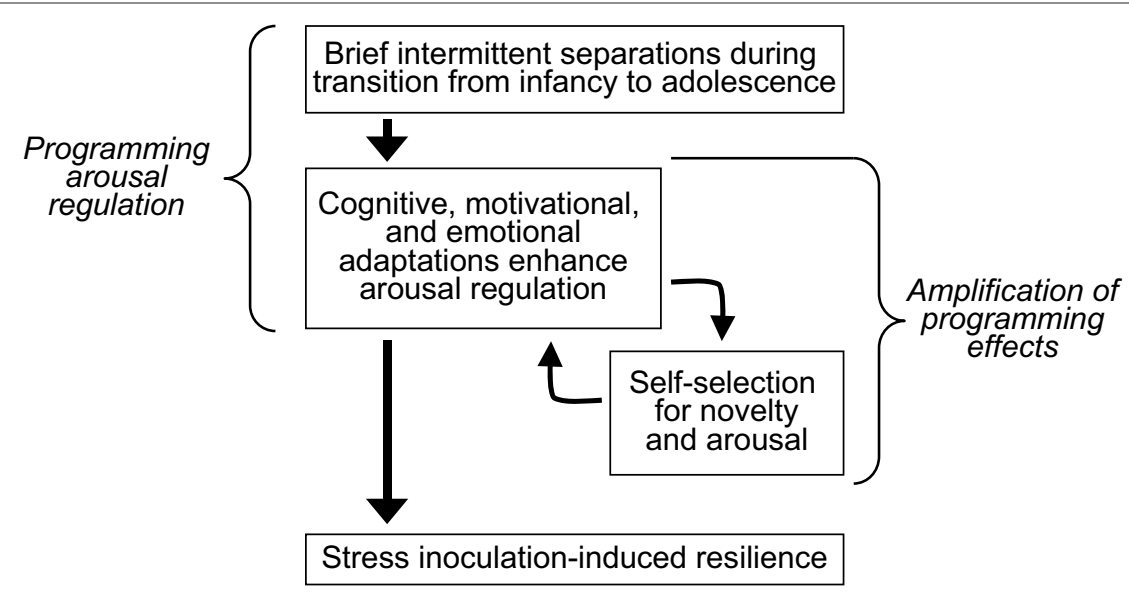

FIGURE 1 | Developmental cascade model of stress inoculation-induced resilience. Coping with brief intermittent separations that simulate a naturally occurring but stressful condition enhances arousal regulation which promotes curiosity and thereby amplifies the initial effects induced by coping with stress. be adaptive in adolescence and adulthood when animals reside in environments that are similar to the one in which they are born (Cameron et al., 2005). Programming effects are not necessarily fixed, however, as subsequent cascades linking multiple domains of behavior across life span development may amplify (or diminish) the initial effects induced by the process of coping with early life stress. Cascade effects in life span models of development are increasingly viewed as important in humans (Schaffer, 2000; Burt et al., 2008) but are seldom considered in animal behavior research (O’Connor and Cameron, 2006).

\section{PREFRONTAL NEUROPLASTICITY AND RESILIENCE}

Arousal regulation, cognitive control, and curiosity are mediated, in part, by prefrontal cortical brain regions in human and nonhuman primates (Price, 2005; Barbas and Zikopoulos, 2007). Therefore, we used non-invasive neuroimaging to examine prefrontal development at 3.3 years of age (Katz et al., 2009). In keeping with previous observations (Lyons et al., 2002) we found that monkeys exposed to intermittent separations had larger ventromedial but not larger dorsolateral prefrontal cortical volumes compared to monkeys not exposed to prior intermittent separations. Larger volumes do not reflect increased cortical thickness but instead represent surface area expansion of ventromedial prefrontal cortex (Katz et al., 2009).

These findings are of interest because ventromedial prefrontal cortical size in humans predicts diminished impulsivity (Matsuo et al., 2009), lower harm avoidance (Yamasue et al., 2008), and greater retention of learned extinction of fear (Milad et al., 2005). Recent neuroimaging studies of humans support results from animal research confirming that learned extinction of fear is mediated by prefrontal downregulation of arousal via inhibitory connections that diminish neural output from the amygdala (Delgado et al., 2008; Quirk and Mueller, 2008). Additional evidence likewise suggests that differences in the balance between top-down prefrontal regulation and arousal-inducing amygdala activation may account for trait-like differences in successfully coping with stress (Bishop, 2007; Drabant et al., 2009).

Despite evidence that coping with stress depends on myelinated prefrontal cortical and subcortical (e.g., amygdala)interconnections, myelination is not often included in discussions of neural plasticity as a mechanism for experience-dependent arousal regulation and resilience. In the study described above we used diffusion tensor imaging to examine prefrontal myelination in terms of fractional anisotropy at 3.3 years of age (Katz et al., 2009). Increased fractional anisotropy occurs when tissue microstructure constrains water proton diffusion directionality as exemplified by myelination of axons in white matter. Myelination increases nerve conduction velocities and facilitates synchronous firing of neurons by reducing travel distance effects in distributed networks (Fields, 2008). Coordination of firing inputs to maximize temporal summation at postsynaptic neurons is the foundation for a key concept in neuroplasticity and development - neurons that fire together, wire together. In children and adolescents, myelination of prefrontal interconnections determined by fractional anisotropy increases with age (BarneaGoraly et al., 2005) and maturation of prefrontal-dependent functions (Casey et al., 2007).

In monkeys exposure to intermittent separations increases ventromedial but not dorsolateral prefrontal white matter measures of fractional anisotropy (Katz et al., 2009). This difference coincides with the difference in ventromedial and not dorsolateral prefrontal cortical volumes measured in the same sample of monkeys. White matter measures of fractional anisotropy in ventromedial but not dorsolateral regions correlate with their respective volumes of prefrontal cortex (Katz et al., 2009). Taken together these findings suggest that the process of coping with early life stress increases prefrontal myelination and expands a region of cortex that broadly controls arousal regulation and resilience.

The effect size for these neuroadaptations is small but similar in magnitude to that observed for environmental enrichment in marmoset monkeys (Kozorovitskiy et al., 2005) and rats (Rosenzweig, 2003). Enrichment entails repeated exposure to novel inanimate and/or social stimulation (Fox et al., 2006) and appears to elicit neuroendocrine indications of mild stress in rats (Moncek et al., 2004). After exposure to enrichment, however, rats show diminished indications of anxiety, increased exploration, and enhanced prefrontal-dependent learning compared to non-enriched controls (Schrijver et al., 2004; Fox et al., 2006). This evidence from rodents 
combined with our primate findings suggest that enrichment effects may be mediated, in part, by the process of coping with stress.

\section{STRESS INOCULATION OR MATERNAL MEDIATION?}

Brief intermittent exposure to foot shock during infancy enhances arousal regulation and resilience in rats (Levine, 1962). Evidently, this outcome reflects the effects of increased maternal stimulation received after foot shock when rat pups are returned to the nest (Smotherman and Bell, 1980). That increased maternal stimulation enhances arousal regulation is supported by evidence that licking, grooming, and arched-back nursing correlate inversely with HPA axis activation in pups studied as adults (Cameron et al., 2005).

To determine whether separation effects in monkeys are maternally mediated, maternal behavior and cortisol levels were examined in monkeys randomized to three postnatal conditions (Parker et al., 2006). In one condition, each monkey was separated from its mother and the natal group for 10 weekly sessions that each lasted $1 \mathrm{~h}$ in duration. In the second condition, each monkey and its mother were removed together as a pair and separated from the natal group for 10 weekly $1 \mathrm{~h}$ sessions. Both of these social separation conditions elicit locomotor agitation, distress vocalizations, and HPA axis activation with baseline measures of arousal restored soon after reunion with members of the natal group (Coe et al., 1983; Jordan et al., 1985). In the third condition, non-separated monkeys were raised in undisturbed groups.

Rearing-related differences in maternal behavior did not correspond with differences in the development of arousal regulation. Monkeys exposed along with their mother to intermittent separations received less maternal care in the home cage, yet both intermittent separation conditions enhanced arousal regulation inferred from diminished stress-levels of cortisol compared to the levels measured in monkeys raised in undisturbed groups (Parker et al., 2006). These and related findings suggest that changes in arousal regulation more closely correspond to stress exposure than to separation-induced changes in maternal care. Similar conclusions have been reported in recent investigations of early life stress and developmental plasticity in rats (Macri and Wurbel, 2006; Tang et al., 2006).

\section{ENHANCING RESILIENCE IN HUMANS}

Controlled exposure to stress-related cues is a key feature of resiliency training for people that work in conditions where performance in the face of adversity is required, e.g., medical and military personnel, aviators, police, firefighters, and rescue workers (Meichenbaum, 2007; Stetz et al., 2007). A similar process also occurs during cognitive behavior exposure therapy for stress-induced psychopathology. Patients are taught to imagine a graded series of stressful situations relevant to their particular condition, and then to interact in vivo with these relevant stressful situations. Repeated exposure to relevant stressors is thought to activate cognitive and emotional processing within and between exposure sessions, and thereby modify erroneous conditions that underlie the disorder (Foa and Kozak, 1986). A critical feature of this approach is the idea that simultaneous cognitive and emotional processing is required for interventionbased changes in behavior to occur (De Raedt, 2006).

Although exposure therapy for patients and resiliency training for healthy humans are administered by professional psychologists and psychiatrists, results from our studies of monkeys support
Epstein's (1983) suggestion that these interventions build on a process that commonly occurs spontaneously without formal guidance. A better understanding of this process may help to enhance interventions designed to improve human mental health.

Consider, for example, the role of habituation to stressors in exposure therapy. Within-session habituation is thought to reflect the cognitive and emotional processing required to achieve adaptive long-term changes in behavior (Foa and Kozak, 1986). As a consequence, the duration of each session has generally ranged from 45 to $120 \mathrm{~min}$. Recently, however, a trial of shorter 30-min exposure sessions has raised the possibility that between- and not within-session habituation effectively reduces anxiety (van Minnen and Foa, 2006). Animal studies designed to compare within- and between-session habituation as predictors of stress inoculation effects may provide relevant and clinically useful information. A better understanding of this issue is needed because of concerns that prolonged exposure sessions may cause patient dropout from therapy and impede recovery (Hembree et al., 2003).

Further research is likewise needed to determine whether medications aid or interfere with recovery from stress-induced psychopathology. Benzodiazapines have been prescribed to immediately reduce anxiety in humans after exposure to severe forms of stress, but placebo controlled studies have failed to demonstrate shortor long-term efficacy (Braun et al., 1990; Gelpin et al., 1996). In some studies, early administration of benzodiazapines have actually been associated with less favorable outcomes (Braun et al., 1990; Mellman et al., 2002). If simultaneous cognitive and emotional processing of stressors is required to achieve long-term adaptations, then drugs that interfere with these processes may impede or prevent recovery. In animal models, benzodiazepines impair learned extinction of conditioned fear (Bouton et al., 1990) and similar studies are needed to determine whether benzodiazapines interfer with the development of stress inoculation-induced resilience.

\section{CONCLUSIONS}

In this review, we have discussed aspects of stress neurobiology that warrant greater attention in behavioral neuroscience research. Although stress is commonly viewed as having deleterious effects, the process of coping with stress is also considered a potential mediating mechanism for the positive effects induced in laboratory rodents by environmental enrichment (Fox et al., 2006). Controlled exposure to stress-related cues is a key feature of resiliency training for healthy humans (Meichenbaum, 2007; Stetz et al., 2007) and is exploited in therapeutic interventions for patients with mood and anxiety disorders (Foa and Kozak, 1986; De Raedt, 2006). In monkeys, myelination and prefrontal cortical expansion induced by coping with brief intermittent separations that simulate a naturally occurring but stressful experience supports transformations in multiple domains of behavior and physiology (Lyons et al., 1999; Levine and Mody, 2003; Parker et al., 2004, 2005, 2007; Katz et al., 2009). Stress is an inevitable aspect of life that cannot be eliminated or avoided for very long. To consider stress solely as inherently destructive overlooks its broader role in shaping adaptive outcomes in the course of life span development.

\section{ACKNOWLEDGMENTS}

Supported by Public Health Service grants MH47573, MH66537, MH77884, and DA16902. 


\section{REFERENCES}

Andrews, M. W., and Rosenblum, L. A. (1993). Assessment of attachment in differentially reared infant monkeys (Macaca radiata): response to separation and a novel environment. J. Comp. Psychol. 107, 84-90.

Barbas, H., and Zikopoulos, B. (2007). The prefrontal cortex and flexible behavior. Neuroscientist 13, 532-545.

Barnea-Goraly, N., Menon, V., Eckert, M., Tamm,L., Bammer,R., Karchemskiy, A., Dant, C. C., and Reiss, A. L. (2005). White matter development during childhood and adolescence: a crosssectional diffusion tensor imaging study. Cereb. Cortex 15, 1848-1854.

Bishop, S. J. (2007). Neurocognitive mechanisms of anxiety: an integrative account. Trends Cogn. Sci. 11, 307-316.

Boinski, S., and Fragaszy, D. M. (1989). The ontogeny of foraging in squirrel monkeys, Saimiri oerstedi. Anim. Behav. 37, 415-428.

Bouton, M. E., Kenney, F. A., and Rosengard, C. (1990). State-dependent fear extinction with two benzodiazepine tranquilizers. Behav. Neurosci. 104, 44-55.

Brady, A. G. (2000). Research techniques for the squirrel monkey (Saimiri). ILAR J. 41, 10-18.

Braun, P., Greenberg, D., Dasberg, H., and Lerer, B. (1990). Core symptoms of posttraumatic stress disorder unimproved by alprazolam treatment. J. Clin. Psychiatry 51, 236-238.

Burt, K. B., Obradovic, J., Long, J. D., and Masten, A. S. (2008). The interplay of social competence and psychopathology over 20 years: testing transactional and cascade models. Child Dev. 79, 359-374.

Cameron, N. M., Champagne, F. A., Parent, C., Fish, E. W., OzakiKuroda, K., and Meaney, M. J. (2005). The programming of individual differences in defensive responses and reproductive strategies in the rat through variations in maternal care. Neurosci. Biobehav. Rev. 29, 843-865.

Casey, B. J., Epstein, J. N., Buhle, J., Liston, C., Davidson, M.C., Tonev, S. T., Spicer, J., Niogi, S., Millner, A. J., Reiss, A., Garrett, A., Hinshaw, S. P., Greenhill, L. L., Shafritz, K. M., Vitolo, A., Kotler, L.A., Jarrett,M.A., and Glover, G. (2007). Frontostriatal connectivity and its role in cognitive control in parent-child dyads with ADHD. Am. J. Psychiatry 164, 1729-1736.

Coe, C. L., Glass, J. C., Wiener, S. G., and Levine, S. (1983). Behavioral, but not physiological, adaptation to repeated separation in mother and infant primates. Psychoneuroendocrinology 8, 401-409.
De Raedt, R. (2006). Does neuroscience hold promise for the further development of behavior therapy? The case of emotional change after exposure in anxiety and depression. Scand. J. Psychol. 47, 225-236.

Delgado, M. R., Nearing, K. I.,Ledoux, J. E., and Phelps, E. A. (2008). Neural circuitry underlying the regulation of conditioned fear and its relation to extinction. Neuron 59, 829-838.

Dettling, A. C., Feldon, J., and Pryce, C. R. (2002). Repeated parental deprivation in the infant common marmoset (Callithrix jacchus, primates) and analysis of its effects on early development. Biol. Psychiatry 52, 1037-1046.

Diamond,A. (1990). Developmental time course in human infants and infant monkeys, and the neural bases of, inhibitory control of reaching. Ann. N. Y. Acad. Sci. 608, 637-676.

Diamond, A., Zola-Morgan, S., and Squire, L. R. (1989). Successful performance by monkeys with lesions of the hippocampal formation on $\mathrm{AB}$ and object retrieval, two tasks that mark developmental changes in human infants. Behav. Neurosci. 103, 526-537.

Dias, R., Robbins, T.W., and Roberts, A. C. (1996). Primate analogue of the Wisconsin card sorting test: effects of excitotoxic lesions of the prefrontal cortex in the marmoset. Behav. Neurosci. 110, 872-886.

Dienstbier, R. A. (1989). Arousal and physiological toughness: implications for mental and physical health. Psychol. Rev. 96, 84-100.

Drabant, E. M., McRae, K., Manuck, S. B., Hariri, A. R., and Gross, J. J. (2009). Individual differences in typical reappraisal use predict amygdala and prefrontal responses. Biol. Psychiatry 65, 367-373.

Ellis, B. J., Essex, M. J., and Boyce, W. T. (2005). Biological sensitivity to context: II. Empirical explorations of an evolutionary-developmental theory. Dev. Psychopathol. 17, 303-328.

Epstein, S. (1983). Natural healing processes of the mind: graded stress inoculation as an inherent coping mechanism. In Stress Reduction and Prevention, D. Meichenbaum, and M. E. Jaremko, eds (New York, Plenum Press), pp. 39-66.

Fields, R. D. (2008). White matter in learning, cognition and psychiatric disorders. Trends Neurosci. 31, 361-370.

Foa, E. B., and Kozak, M. J. (1986). Emotional processing of fear: exposure to corrective information. Psychol. Bull. 99, 20-35.

Fox, C., Merali, Z., and Harrison, C. (2006). Therapeutic and protective effect of environmental enrichment against psychogenic and neurogenic stress. Behav. Brain Res. 175, 1-8.

Garmezy, N., Masten,A.S., and Tellegen, A (1984). The study of stress and competence in children: a building block for developmental psychopathology. Child Dev. 55, 97-111.

Gelpin, E., Bonne, O., Peri, T., Brandes, D., and Shalev, A. Y. (1996). Treatment of recent trauma survivors with benzodiazepines: a prospective study. J. Clin Psychiatry 57, 390-394.

Gibson, E. J. (1988). Exploratory behavior in the development of perceiving, acting, and the acquiring of knowledge. Annu. Rev. Psychol. 39, 1-41.

Gunnar, M. R., Frenn, K., Wewerka, S. S. and Van Ryzin, M. J. (2009). Moderate versus severe early life stress: associations with stress reactivity and regulation in 10-12-year-old children. Psychoneuroendocrinology 34, 62-75.

Hembree, E. A., Foa, E. B., Dorfan, N. M., Street, G. P., Kowalski, J., and Tu, X. (2003). Do patients drop out prematurely from exposure therapy for PTSD? J. Trauma. Stress 16, 555-562.

Hennessy, M. B. (1986). Multiple, brief maternal separations in the squirrel monkey: changes in hormonal and behavioral responsiveness. Physiol. Behav. 36, 245-250.

Hinde, R. A., and Spencer-Booth, Y. (1971). Effects of brief separation from mother on rhesus monkeys. Science 173, 111-118.

Hughes, R. N. (2007). Neotic preferences in laboratory rodents: issues, assessment and substrates. Neurosci. Biobehav. Rev. 31, 441-464.

Jordan, T. C., Hennessy, M. B. Gonzalez, C. A., and Levine, S. (1985). Social and environmental factors influencing mother-infant separation-reunion in squirrel monkeys. Physiol. Behav. 34, 489-493.

Katz, M., Liu, C., Schaer, M., Parker, K. J., Ottet, M., Epps, A., Buckmaster, C. L., Bammer, R. L., Moseley, M. E., Schatzberg, A. F., Eliez, S., and Lyons, D. M. (2009). Prefrontal plasticity and stress inoculation-induced resilience. Dev. Neurosci. 31, 293-299.

Khoshaba, D. M., and Maddi, S. R. (1999) Early experiences in hardiness development. Consult. Psychol. J. Prac. Res. 51, 106-116.

Kozorovitskiy, Y., Gross, C. G., Kopil, C., Battaglia, L., McBreen, M., Stranahan, A. M., and Gould, E. (2005). Experience induces structural and biochemical changes in the adult primate brain. Proc. Natl. Acad. Sci. U.S.A. 102, 17478-17482.

Levine, S. (1962). The effects of infantile experience on adult behavior. In Experimental Foundations of Clinical
Psychology, A. J. Bachrach, ed. (New York, Basic Books), pp. 139-169.

Levine, S., and Mody, T. (2003). The longterm psychobiological consequences of intermittent postnatal separation in the squirrel monkey. Neurosci. Biobehav. Rev. 27, 83-89.

Lyons, D. M., Afarian, H., Schatzberg, A. F., Sawyer-Glover, A., and Moseley, M. E. (2002). Experience-dependent asymmetric variation in primate prefrontal morphology. Behav. Brain Res. 136, 51-59.

Lyons, D. M., Kim, S., Schatzberg, A. F., and Levine, S. (1998). Postnatal foraging demands alter adrenocortical activity and psychosocial development. Dev. Psychobiol. 32, 285-291.

Lyons, D. M., Lopez, J. M., Yang, C., and Schatzberg, A. F. (2000a). Stress-level cortisol treatment impairs inhibitory control of behavior in monkeys. J. Neurosci. 20, 7816-7821.

Lyons, D. M., Martel, F. L., Levine, S., Risch, N. J., and Schatzberg, A. F. (1999). Postnatal experiences and genetic effects on squirrel monkey social affinities and emotional distress. Horm. Behav. 36, 266-275.

Lyons, D. M., and Parker, K. J. (2007). Stress inoculation-induced indications of resilience in monkeys. J. Trauma. Stress 20, 423-433.

Lyons, D. M., Yang, C., Mobley, B. W., Nickerson, J. T., and Schatzberg, A. F. (2000b). Early environmental regulation of glucocorticoid feedback sensitivity in young adult monkeys. J. Neuroendocrinol. 12, 723-728.

Macri, S., and Wurbel, H. (2006). Developmental plasticity of HPA and fear responses in rats: a critical review of the maternal mediation hypothesis. Horm. Behav. 50, 667-680.

Mason, W. A. (1971). Motivational factors in psychosocial development. In Nebraska Symposium on Motivation, W. J. Arnold, and M. M. Page, eds (Lincoln, University of Nebraska Press), pp. 35-67.

Matsuo, K., Nicoletti, M., Nemoto, K., Hatch, J. P., Peluso, M. A., Nery, F. G., and Soares, J. C. (2009). A voxel-based morphometry study of frontal gray matter correlates of impulsivity. Hum. Brain Mapp. 30, 1188-1195.

Meichenbaum, D. (2007). Stress inoculation training: a preventative and treatment approach. In Principles and Practice of Stress Management, P. M. Lehrer, et al., eds (New York, N.Y., Guildford Press), pp. 497-518.

Mellman, T.A., Bustamante, V., David, D., and Fins, A. I. (2002). Hypnotic medication in the aftermath of trauma. J. Clin. Psychiatry 63, 1183-1184.

Milad, M. R., Quinn, B. T., Pitman, R. K., Orr, S. P., Fischl, B., and Rauch, S. L. 
(2005). Thickness of ventromedial prefrontal cortex in humans is correlated with extinction memory. Proc. Natl. Acad. Sci. U.S.A. 102, 10706-10711.

Moncek, F., Duncko, R., Johansson, B. B., and Jezova, D. (2004). Effect of environmental enrichment on stress related systems in rats. J. Neuroendocrinol. 16, 423-431.

Mortimer, J. T., and Staff, J. (2004). Early work as a source of developmental discontinuity during the transition to adulthood. Dev. Psychopathol. 16, 1047-1070.

O'Connor, T. G., and Cameron, J. L. (2006). Translating research findings on early experience to prevention: animal and human evidence on early attachment relationships. Am. J. Prev. Med. 31, S175-S181.

Parker, K. J., Buckmaster, C.L., Justus, K. R., Schatzberg, A. F., and Lyons, D. M. (2005). Mild early life stress enhances prefrontal-dependent response inhibition in monkeys. Biol. Psychiatry 57, 848-855.

Parker, K. J., Buckmaster, C. L., Schatzberg, A. F., and Lyons, D. M. (2004). Prospective investigation of stress inoculation in young monkeys. Arch. Gen. Psychiatry 61, 933-941.

Parker, K. J.,Buckmaster, C.L.,Sundlass, K., Schatzberg, A. F., and Lyons, D. M. (2006). Maternal mediation, stress inoculation, and the development of neuroendocrine stress resistance in primates. Proc. Natl. Acad. Sci. U.S.A. 103, 3000-3005.

Parker, K. J., Rainwater, K. L., Buckmaster, C. L., Schatzberg, A. F.,
Lindley, S. E., and Lyons, D. M. (2007). Early life stress and novelty seeking behavior in adolescent monkeys. Psychoneuroendocrinology 32, 785-792.

Price, J. L. (2005). Free will versus survival: brain systems that underlie intrinsic constraints on behavior. J. Comp. Neurol. 493, 132-139.

Pryce, C. R., Dettling, A., Spengler, M., Spaete, C., and Feldon, J. (2004). Evidence for altered monoamine activity and emotional and cognitive disturbance in marmoset monkeys exposed to early life stress. Ann. N. Y. Acad. Sci. 1032, 245-249.

Quirk, G. J., and Mueller, D. (2008). Neural mechanisms of extinction learning and retrieval. Neuropsychopharmacology 33, 56-72.

Roder, E. L., Timmermans, P. J., and Vossen, J. M. (1989). Effects of rearing and exposure condition upon the acquisition of phobic behaviour in cynomolgus monkeys. Behav. Res. Ther. 27, 221-231.

Rosenzweig, M. R. (2003). Effects of differential experience on the brain and behavior. Dev. Neuropsychol. 24, 523-540.

Rutter, M. (2006). Implications of resilience concepts for scientific understanding. Ann. N. Y. Acad. Sci. 1094, $1-12$.

Sackett, G. P. (1972). Exploratory behavior of rhesus monkeys as a function of rearing conditions and sex. Dev. Psychol. 6, 260-270.

Sanchez, M. M., Ladd, C. O., and Plotsky, P. M. (2001). Early adverse experience as a developmental risk factor for later psychopathology: evidence from rodent and primate models. Dev. Psychopathol. 13, 419-449.

Schaffer, H. R. (2000). The early experience assumption: past, present, and future. Int. J. Behav. Dev. 24, 5-14.

Schrijver, N. C., Pallier, P. N., Brown, V. J. and Wurbel, H. (2004). Double dissociation of social and environmental stimulation on spatial learning and reversal learning in rats. Behav. Brain Res. 152, 307-314.

Silvia, P. J. (2008). Interest - the curious emotion. Curr. Dir. Psychol. Sci. 17 57-60.

Smotherman, W. P., and Bell, R. W. (1980). Maternal mediation of early experiences. In Maternal Influences and Early Behavior, W.P. Smotherman and R. W. Bell, eds (New York, Spectrum Publications)

Stetz, M. C., Thomas, M. L., Russo, M. B. Stetz, T. A., Wildzunas, R. M. McDonald, J. J., Wiederhold, B. K. and Romano, J. A., Jr. (2007). Stress, mental health, and cognition: a brief review of relationships and countermeasures. Aviat. Space Environ. Med. 78, B252-B260.

Tang, A. C., Akers, K. G., Reeb, B. C. Romeo, R. D., and McEwen, B. S. (2006). Programming social, cognitive, and neuroendocrine development by early exposure to novelty. Proc. Natl. Acad. Sci. U.S.A. 103, 15716-15721.

van Minnen, A., and Foa, E. B. (2006). The effect of imaginal exposure length on outcome of treatment for PTSD. J. Trauma. Stress 19, 427-438.
Wallis, J. D., Dias, R., Robbins, T. W., and Roberts, A. C. (2001). Dissociable contributions of the orbitofrontal and lateral prefrontal cortex of the marmoset to performance on a detour reaching task. Eur. J. Neurosci. 13, 1797-1808.

Yamasue, H., Abe, O., Suga, M. Yamada, H., Inoue, H., Tochigi, M., Rogers, M., Aoki, S., Kato, N., and Kasai, K. (2008). Gender-common and -specific neuroanatomical basis of human anxiety-related personality traits. Cereb. Cortex 18, 46-52.

Conflict of Interest Statement: The research reviewed in this report was conducted in the absence of any commercial or financial relationships that could be construed as a potential conflict of interest.

Received: 01 May 2009; paper pending published: 10 July 2009; accepted: 01 September 2009; published online: 18 September 2009.

Citation: Lyons DM, Parker KJ, Katz $M$ and Schatzberg AF (2009) Developmental cascades linking stress inoculation, arousal regulation, and resilience. Front. Behav. Neurosci. 3:32. doi: 10.3389/neuro.08.032.2009

Copyright (C) 2009 Lyons, Parker, Katz and Schatzberg. This is an open-access article subject to an exclusive license agreement between the authors and the Frontiers Research Foundation, which permits unrestricted use, distribution, and reproduction in any medium, provided the original authors and source are credited. 